\title{
New Development of the new Period Erhu Music and Playing Skills
}

\author{
Jing Shi \\ XingTai University, Xingtai, 054001, China
}

Keywords: New period, Erhu music, Performance skills,Composing techniques

\begin{abstract}
Many erhu works with modern consciousness appeared in serious music field at the end of the last century, the Erhu playing skills in the works was received attention. Erhu music development space can be expanded and diversified development pattern gradually formed. This paper put forward development of erhu music types through discussion of erhu music works in the new period, in view of the new period playing technique and skills development, in order to provide certain reference for the industry.
\end{abstract}

\section{Introduction}

The new period social development trend is diversified, the alarming spreading rate also make contemporary erhu music and the performing skill present a new pattern. Erhu as a musical instrument,the maximum value is nothing but new erhu works produced with the new playing style appeared. The author in recent years appear professional erhu works with modern to create awareness of the playing techniques are analyzed. In order to promote the erhu music in the field of serious music better development. And the development direction of the new things inevitable based on the social direction, the pluralistic social environment doomed with erhu music will be in line with the needs of the public to explore new era of erhu music and playing skills also has the important meaning.

\section{Overview of erhu music in New Period}

At the beginning of this century, erhu music has been widespread concern from all walks of life, in the field of creation of a number of new works. The authors of traditional erhu works were adapted or created, in order to maximize the fit between the contemporary thoughts and feelings, most of the works are applied modern consciousness creative approach and creative consciousness. During this period the urheen composer mostly have a certain professional background, overall quality compared to the past has greatly improved, the diversification trend of erhu music creation techniques and musical language of expression. During this period the erhu works for the playing skills to indicate the direction. Among them, the work can be divided into two categories:

\section{Arrangement}

"The shining Tashikuergan", "Carmen fantasy ", " Infinite motion " works more suitable for erhu, because elements such works mostly come to the violin , the advantages of stringed instruments of it make it has common on performance and tuning with erhu. Therefore, it' s not easy for the adaption of the traditional composer . But close to copy after all, there is a world of difference, alien to many violin composition skills and playing techniques and erhu, erhu in part of the range is difficult to do effect. So the traditional arrangement were the second creation process is not easy, requires a combination of original thought and the erhu instrument emotional expression. Under normal circumstances, meeting the erhu register, adapted generally take octave conversion or transpose techniques, encountered many dual tone playing adaptations generally take the melody voice method, uncoordinated bowing problems encountered, adapted generally use new bowing alternative, all adaptation follows the original inner emotional expression of the principles, although there are many factors that affect the process of adaptation to consider, but instruments timbre and sound quality issues bear the brunt. Listen carefully to the identification of two kinds of stringed musical instruments adapted works, will find the essential difference of timbre. So for adaptation of the selection of works should be close to the erhu timbre and emotional characteristics, ignoring the 
difference of timbre, a free adaptation of instruments is likely to mislead the public's perception of erhu music language.

\section{Performance}

The "Carmen theme fantasy " is a typical representative of the performance of the adapted music, the music shows a variety of performance skills and the Comprehensive music quality of the composer. In real life, some adaptations tend to grasp clear Erhu melody point to find the most suitable for the performance, they take the most direct way to present his work in front of people, easy and mass produce emotional resonance. Show strong technology laid the foundation for the later creation of the composer, no longer tied to the tune of traditional erhu play mode. "The shining Tashikuergan" is the typical one, different adaptation techniques make the works showing a different erhu tonality, Yan Jiemin adaptation and Taiwan Song Contest version is adapted version of the current relatively common, very good performance characteristics of the original. The Taiwan Song Contest version less difficult and popularization rate is higher, after the adaptation of the additional introduction can be divided into warm dance and singing heartily in two parts, can also according to presto, Allegro, powder plate, plate, the theme of refinement into five parts. Tajik nationality mode and fast playing skills enable erhu after the adaptation of the musical image changing, erhu playing the whole frame of space gets a promotion, the gained popularity, in line with the overall situation of the development of modern music, that mass this songs impression even stay at the level of erhu, rather than violin works.

\section{The emergence and development of the techniques of playing in the new period}

The 21st century is Chinese national instrumental music 's key period of vigorous development of skills, virtuoso playing style swept the world. There has been a large number of different voices of modernist music, instrumental music began has received widespread attention from the people. In the last century at the end of this century and at the beginning of the period, a variety of Erhu playing skills show in front of people, accompanied by a large number of erhu works produced, playing skills have been greatly improved. Composer in the complicated composition skills before seriously consider the original emotion expression, avoid creation becomes a pile of simple techniques, sound technical innovation also promote composer continue to study new techniques to adapt, erhu music playing skills showing the two development model of general development and new techniques. In this paper the author for general skills development applied to probe into and its basic right slow Longbow play, for example, by changing the rhythm power etc. to enrich the techniques, and appropriate to break conventional playing skills to play the effect.

The emergence of the new sound is the goal of the modern composer's unremitting pursuit. To achieve this goal, it is necessary to break some tradition and create some new techniques. For example in some contemporary composers erhu monotonic horizontal lines play has been very difficult to meet its longitudinal or acoustic requirements, there will be some new techniques to make up for the demand, " Double string performance " is a typical case. When playing in the process, will be different because of their subjective consciousness, required to achieve the effect of a play and the audience, two distinct emerge as the times require playing style: one is the music playing method, this way of playing the general volume smaller, speed of slow, must put the bow hair in compressed two string between, let the bow hair can reach two string friction effect, the playing techniques are often applied in performance in the illusory adagio; playing method of noise, this technique is mainly used in the bow string arch bar with the mullahs, wherein the volume is high, clean, sound quality is a typical feature of the different playing techniques, have the unique role of dynamic scenes and characters of inner conflict expression. In addition, the right hand percussion bow, shoot the shell, the left hand curved column method and other different techniques are also new performance skills in the representative.

In the new period the birth of erhu music professional works will have a lot of basic etudes as paving the way, the player needs these etudes to solve some difficult technical problems and deepen the playing techniques and basic understanding. Because the renewal of music content and form of 
the music inevitable requirement musical skills synchronization techniques improve also must react on the development of erhu music. Is summarized by the author can be found the playing techniques mainly include: large changes in the sound and variable interval, to high the notes application, complex rhythms and complex rhythms of high difficulty combination.

\section{Development types of erhu music in New Period}

In the new period development of world music show diversified pattern, music gradually get rid of the bound of traditional creation system, music language and image more full of mystery and traditional tonality to be a breakthrough, melody began to board the stage of history, become a key point of the combination of intervals and interval relations, twelve tone system and serial music and new music have also makes composing theory system further. In the new period, our country and the world more closely linked, the contemporary composer and the world music stage distance is close, the work also has the bright time feeling. Based on the early 1980s works, such as "red plum Capriccio", "the Great Wall Capriccio", new era of erhu music will continue to the modernist music system near, multiple techniques of performance characteristics significantly. But due to the system of modern music exploration is not yet mature, music form many aspects show is still not perfect, so the public contact time and the number of new works is very limited, struggling with the development of modern music, this kind of phenomenon in the field of erhu is still widespread. But with the deepening of network development and international communication, the mass ideology progress, artistic accomplishment promotion, accept new things faster, the attention of erhu music and the performing skill there will be more and more. Among them, the type of development of erhu music works in the new period:

\section{Traditional music creation technique still holds the dominant position}

"The first Erhu Concerto "and the" Royal "is the typical representative of this kind of work, there are some listeners, works with a distinctive theme color, soft melody, unified in a traditional formal framework in the request. "First Erhu Concerto" at the end of the last century was known as the reason is although its creation in earlier, but the doping the many modern art technique of expression, such as taking ang modulation and tonality free, irregular rhythm changes. This works based on the concerto form changed the idea of the people the value of traditional erhu, breaking the erhu single playing the melody of tones limits, expanding the erhu in the field of pure music performance, with abundant artistic potential. Music in three movements of ups and downs, not blindly use traditional folk tone expression, melodic and do not deliberately, composer of Erhu Chinese flavor began integration of a variety of elements of style, this is the erhu melody work innovation is a big step. Show a piece of a variety is not regular and rhythmic and complex rhythm, accentual rhythm has been broken in the reorganization of the work, many liaisons and endless application that works better presentation, Untitled work there but also to the creation of Erhu has a more profound social themes. Especially I think Hong Mei version, the performance is more thorough. This work is of significance, but also because of the use of the traditional techniques of the tone of the larger, so the author believes that some of the traditional music creation techniques are still dominant.

\section{Relative balance proportion works both on traditional composing techniques and modernist techniques}

"The Tianshan style "," Rhapsody "," concerto " are the typical representative of this kind of works, composers began to find that really belongs to the erhu sound, and the sound is similar to the violin and other instruments which can not be replaced, such as more texture and powerful timbre, delicate euphemism and fusion of personality and so on. "Rhapsody" works did not use large musical structure, but the tone of the nation were tonal transform processing, makes the work not because of different passages become boring, mellow clang, flexible naughty multiple transformation make up the blank of creation and unique to the erhu music features can be displayed. For the rich minority ethnic color rhythm and interval, this technique can works is compatible with modern music style and national flavor. Beat the lute cylinder knock arch bar, irregular musical harmonies, let the creative 
skills of Erhu gradually getting rid of the traditional imprisonment, the piece from multiple angles close to the requirements of modern music style.

\section{The works with the detective factor of the modern music creation technique}

Erhu and Orchestra "Fire Festival", erhu and dulcimer "double Que" are typical representative of this kind of works, during this time the Chinese composers, many foreign composers of other national style of music, erhu and a variety of musical ensemble, erhu and multiple band or the voice combination play different, many more overwhelmed. Modern style of composing technique has become the main writing techniques in such works, increase the intonation, rhythm to break the rhythm, the singular tone is the typical techniques. Which can better show the musician inherent' music understanding to the to contemporary musical personality, not satisfied with traditional music expression. These works are mostly used to show the erhu is a very mysterious thing. In short, the uncertainty phoneme, the illusory sense of the rhythm, casual sense bring new auditory feast the audience to.

\section{Concluding remarks:}

To sum up, the development of things will follow the pace of development of the times, even some things with the benefit of hindsight, it must be towards a given direction moving forward circuitously. The direction of development of new things based on the social direction, diversification of social environment destined to the development track of erhu music, whether it is in accordance with the creation of erhu works, or playing skills of expression, if blindly limitations in the traditional technology can not extricate themselves is the inevitable result of the delignification and the needs of the public section. Especially at the beginning of this century, a lot of modern style of the erhu works a surge and current, erhu music compared to traditional music has a greater development space and opportunities. Social overall development situation is good, because of the erhu attributes of musical instrument, new works produced and new playing style is the embodiment of its maximum value. So, on the erhu skills training and education has gradually led to people's attention, which also need to the joint efforts of our practitioners.

\section{References}

[1] Bu Xiaomei. New development of erhu music and playing skills at the end of the 20 century. Journal of Central Conservatory of Music, 2014. 01

[2] Wang Yi, Hu Zhiping. Dual song "river water" and erhu "river water" -- on the adaptation and transplantation reflects the creative. Nanjing Arts Institute Journal (music and performing Edition).2014. 01

[3] Xiao Feng. Talk about intonation problems and solving methods of Erhu. Journal of Xinghai Conservatory of Music. 201302

[4] Sun Yi. Southern Chinese Hu violin runqiang art, dialect and traditional vocal music' effect to southern Chinese Hu violin runqiang art. People's music.2014,(9)

[5] Li Huanzhi, Wang Anguo. Century review, the rheology of the Chinese traditional music and contemporary Chinese music expression. People's music, 2013. (01)

[6] Cao Deben. The Chinese traditional culture and the world multicultural culture. Journal of Tsinghua University (Philosophy and social science edition), 2011. 04 